\title{
Multiple positive solutions for semilinear elliptic systems involving subcritical nonlinearities in $\mathbb{R}^{N}$
}

Huei-li Lin*

\section{${ }^{*}$ Correspondence:}

hlin@mail.cgu.edu.tw

Department of Natural Sciences in

the Center for General Education,

Chang Gung University, Tao-Yuan,

333, Taiwan, R.O.C.

\begin{abstract}
In this paper, we investigate the effect of the coefficient $f(x)$ of the subcritical least $k(\geq 1)$ positive solutions of the semilinear elliptic systems

$$
\begin{cases}-\varepsilon^{2} \Delta \bar{u}+\bar{u}=\lambda g(x)|\bar{u}|^{q-2} \bar{u}+\frac{\alpha}{\alpha+\beta} f(x)|\bar{u}|^{\alpha-2} \bar{u}|\bar{v}|^{\beta} & \text { in } \mathbb{R}^{N} \\ -\varepsilon^{2} \Delta \bar{v}+\bar{v}=\mu h(x)|\bar{v}|^{q-2} \bar{v}+\frac{\beta}{\alpha+\beta} f(x)|\bar{u}|^{\alpha}|\bar{v}|^{\beta-2} \bar{v} & \text { in } \mathbb{R}^{N} \\ \bar{u}, \bar{v} \in H^{1}\left(\mathbb{R}^{N}\right) & \end{cases}
$$
\end{abstract}
nonlinearity. Under some assumptions, for sufficiently small $\varepsilon, \lambda, \mu>0$, there are at

where $\alpha>1, \beta>1,2<q<p=\alpha+\beta<2^{*}=2 N /(N-2)$ for $N \geq 3$.

MSC: $35 J 20 ; 35 J 25 ; 35 J 65$

Keywords: semilinear elliptic systems; subcritical exponents; Nehari manifold

\section{Introduction}

For $N \geq 3, \alpha>1, \beta>1$ and $2<q<p=\alpha+\beta<2^{*}=2 N /(N-2)$, we consider the semilinear elliptic systems

$$
\left\{\begin{array}{l}
-\varepsilon^{2} \Delta \bar{u}+\bar{u}=\lambda g(x)|\bar{u}|^{q-2} \bar{u}+\left.\left.\frac{\alpha}{\alpha+\beta} f(x)|\bar{u}|^{\alpha-2} \bar{u}\right|_{\bar{v}}\right|^{\beta} \quad \text { in } \mathbb{R}^{N} \\
-\varepsilon^{2} \Delta \bar{v}+\bar{v}=\mu h(x)|\bar{v}|^{q-2} \bar{v}+\frac{\beta}{\alpha+\beta} f(x)|\bar{u}|^{\alpha}|\bar{v}|^{\beta-2} \bar{v} \quad \text { in } \mathbb{R}^{N} \\
\bar{u}>0, \quad \bar{v}>0
\end{array} \quad\left(\bar{E}_{\varepsilon, \lambda, \mu}\right)\right.
$$

where $\varepsilon, \lambda, \mu>0$.

Let $f, g$ and $h$ satisfy the following conditions:

(A1) $f$ is a positive continuous function in $\mathbb{R}^{N}$ and $\lim _{|x| \rightarrow \infty} f(x)=f_{\infty}>0$.

(A2) there exist $k$ points $a^{1}, a^{2}, \ldots, a^{k}$ in $\mathbb{R}^{N}$ such that

$$
f\left(a^{i}\right)=\max _{x \in \mathbb{R}^{N}} f(x)=1 \quad \text { for } 1 \leq i \leq k,
$$

and $f_{\infty}<1$.

(A3) $g, h \in L^{m}\left(\mathbb{R}^{N}\right) \cap L^{\infty}\left(\mathbb{R}^{N}\right)$ where $m=(\alpha+\beta) /(\alpha+\beta-q)$, and $g, h \supsetneqq 0$. 
In [1], if $\Omega$ is a smooth and bounded domain in $\mathbb{R}^{N}(N \leq 3)$, they considered the following system:

$$
\begin{cases}\varepsilon^{2} \Delta \bar{u}-\lambda_{1} \bar{u}=\mu_{1} \bar{u}^{3}+\beta \overline{u v}^{2} & \text { in } \Omega ; \\ \varepsilon^{2} \Delta \bar{v}-\lambda_{2} \bar{v}=\mu_{2} \bar{v}^{3}+\beta \bar{u}^{2} \bar{v} & \text { in } \Omega ; \\ \bar{u}>0, \quad \bar{v}>0, & \end{cases}
$$

and proved the existence of a least energy solution in $\Omega$ for sufficiently small $\varepsilon>0$ and $\beta \in\left(-\infty, \beta_{0}\right)$. Lin and Wei also showed that this system has a least energy solution in $\mathbb{R}^{N}$ for $\varepsilon=1$ and $\beta \in\left(0, \beta_{0}\right)$. In this paper, we study the effect of $f(z)$ of $\left(\bar{E}_{\varepsilon, \lambda, \mu}\right)$. Recently, many authors [2-5] considered the elliptic systems with subcritical or critical exponents, and they proved the existence of a least energy positive solution or the existence of at least two positive solutions for these problems. In this paper, we construct the $k$ compact PalaisSmale sequences which are suitably localized in correspondence of $k$ maximum points of $f$. Then we could show that under some assumptions (A1)-(A3), for sufficiently small $\varepsilon, \lambda, \mu>0$, there are at least $k(\geq 1)$ positive solutions of the elliptic system $\left(E_{\varepsilon, \lambda, \mu}\right)$. By the change of variables

$$
x=\varepsilon z, \quad u(z)=\bar{u}(\varepsilon z) \quad \text { and } \quad v(z)=\bar{v}(\varepsilon z)
$$

System $\left(\bar{E}_{\varepsilon, \lambda, \mu}\right)$ is transformed to

$$
\left\{\begin{array}{l}
-\Delta u+u=\lambda g(\varepsilon z)|u|^{q-2} u+\frac{\alpha}{\alpha+\beta} f(\varepsilon z)|u|^{\alpha-2} u|v|^{\beta} \quad \text { in } \mathbb{R}^{N} \\
-\Delta v+v=\mu h(\varepsilon z)|v|^{q-2} v+\frac{\beta}{\alpha+\beta} f(\varepsilon z)|u|^{\alpha}|v|^{\beta-2} v \quad \text { in } \mathbb{R}^{N} ; \\
u>0, \quad v>0 .
\end{array}\right.
$$

Let $H=H^{1}\left(\mathbb{R}^{N}\right) \times H^{1}\left(\mathbb{R}^{N}\right)$ be the space with the standard norm

$$
\|(u, v)\|_{H}=\left[\int_{\mathbb{R}^{N}}\left(|\nabla u|^{2}+u^{2}\right) d z+\int_{\mathbb{R}^{N}}\left(|\nabla v|^{2}+v^{2}\right) d z\right]^{1 / 2} .
$$

Associated with the problem $\left(E_{\varepsilon, \lambda, \mu}\right)$, we consider the $C^{1}$-functional $J_{\varepsilon, \lambda, \mu}$, for $(u, v) \in H$,

$$
\begin{aligned}
J_{\varepsilon, \lambda, \mu}(u, v)= & \frac{1}{2}\|(u, v)\|_{H}^{2}-\frac{1}{\alpha+\beta} \int_{\mathbb{R}^{N}} f(\varepsilon z)|u|^{\alpha}|v|^{\beta} d z \\
& -\frac{1}{q} \int_{\mathbb{R}^{N}}\left(\lambda g(\varepsilon z)|u|^{q}+\mu h(\varepsilon z)|v|^{q}\right) d z .
\end{aligned}
$$

Actually, the weak solution $(u, v) \in H$ of $\left(E_{\varepsilon, \lambda, \mu}\right)$ is the critical point of the functional $J_{\varepsilon, \lambda, \mu}$, that is, $(u, v) \in H$ satisfies

$$
\begin{aligned}
\int_{\mathbb{R}^{N}} & \left(\nabla u \nabla \varphi_{1}+\nabla v \nabla \varphi_{2}+u \varphi_{1}+\nu \varphi_{2}\right) d z \\
& -\lambda \int_{\mathbb{R}^{N}} g(\varepsilon z)|u|^{q-2} u \varphi_{1} d z-\mu \int_{\mathbb{R}^{N}} h(\varepsilon z)|v|^{q-2} \nu \varphi_{2} d z \\
& -\frac{\alpha}{\alpha+\beta} \int_{\mathbb{R}^{N}} f(\varepsilon z)|u|^{\alpha-2} u|v|^{\beta} \varphi_{1} d z-\frac{\beta}{\alpha+\beta} \int_{\mathbb{R}^{N}} f(\varepsilon z)|u|^{\alpha}|v|^{\beta-2} \nu \varphi_{2} d z=0
\end{aligned}
$$

for any $\left(\varphi_{1}, \varphi_{2}\right) \in H$. 
We consider the Nehari manifold

$$
\left.\mathbf{M}_{\varepsilon, \lambda, \mu}=\left\{(u, v) \in H \backslash\{(0,0)\}|| J_{\varepsilon, \lambda, \mu}^{\prime}(u, v),(u, v)\right\rangle=0\right\},
$$

where

$$
\left\langle J_{\varepsilon, \lambda, \mu}^{\prime}(u, v),(u, v)\right\rangle=\|(u, v)\|_{H}^{2}-\int_{\mathbb{R}^{N}} f(\varepsilon z)|u|^{\alpha}|v|^{\beta} d z-\int_{\mathbb{R}^{N}}\left(\lambda g(\varepsilon z)|u|^{q}+\mu h(\varepsilon z)|v|^{q}\right) d z .
$$

The Nehari manifold $\mathbf{M}_{\varepsilon, \lambda, \mu}$ contains all nontrivial weak solutions of $\left(E_{\varepsilon, \lambda, \mu}\right)$.

Let

$$
S_{\alpha, \beta}=\inf _{u, v \in H^{1}\left(\mathbb{R}^{N}\right) \backslash\{(0)\}} \frac{\|(u, v)\|_{H}^{2}}{\left(\int_{\mathbb{R}^{N}}|u|^{\alpha}|v|^{\beta} d z\right)^{2 /(\alpha+\beta)}},
$$

then by [2, Theorem 5], we have

$$
S_{\alpha, \beta}=\left[\left(\frac{\alpha}{\beta}\right)^{\frac{\beta}{\alpha+\beta}}+\left(\frac{\beta}{\alpha}\right)^{\frac{\alpha}{\alpha+\beta}}\right] S_{p},
$$

where $p=\alpha+\beta$ and $S_{p}$ is the best Sobolev constant defined by

$$
S_{p}=\inf _{u \in H^{1}\left(\mathbb{R}^{N}\right) \backslash\{0\}} \frac{\int_{\mathbb{R}^{N}}\left(|\nabla u|^{2}+u^{2}\right) d z}{\left(\int_{\mathbb{R}^{N}}|u|^{p} d z\right)^{2 / p}} .
$$

For the semilinear elliptic systems $(\lambda=\mu=0)$

$$
\left\{\begin{array}{l}
-\Delta u+u=\frac{\alpha}{\alpha+\beta} f(\varepsilon z)|u|^{\alpha-2} u|v|^{\beta} \quad \text { in } \mathbb{R}^{N} \\
-\Delta v+v=\frac{\beta}{\alpha+\beta} f(\varepsilon z)|u|^{\alpha}|v|^{\beta-2} v \quad \text { in } \mathbb{R}^{N} \\
(u, v) \in H
\end{array}\right.
$$

we define the energy functional $I_{\varepsilon}(u, v)=\frac{1}{2}\|(u, v)\|_{H}^{2}-\frac{1}{\alpha+\beta} \int_{\mathbb{R}^{N}} f(\varepsilon z)|u|^{\alpha}|v|^{\beta} d z$, and

$$
\left.\mathbf{N}_{\varepsilon}=\left\{(u, v) \in H \backslash\{(0,0)\}|| I_{\varepsilon}^{\prime}(u, v),(u, v)\right\rangle=0\right\} .
$$

If $f \equiv \max _{z \in \mathbb{R}^{N}} f(z)(=1)$, then we define $I_{\max }(u, v)=\frac{1}{2}\|(u, v)\|_{H}^{2}-\frac{1}{\alpha+\beta} \int_{\mathbb{R}^{N}}|u|^{\alpha}|v|^{\beta} d z$ and

$$
\theta_{\max }=\inf _{(u, v) \in \mathbf{N}_{\max }} I_{\max }(u, v),
$$

where $\mathbf{N}_{\max }=\left\{(u, v) \in H \backslash\{(0,0)\} \mid\left\langle I_{\max }^{\prime}(u, v),(u, v)\right\rangle=0\right\}$.

It is well known that this problem

$$
\left\{\begin{array}{l}
-\Delta u+u=|u|^{p-2} u \quad \text { in } \mathbb{R}^{N} \\
u \in H^{1}\left(\mathbb{R}^{N}\right)
\end{array}\right.
$$

has the unique, radially symmetric and positive ground state solution $w \in H^{1}\left(\mathbb{R}^{N}\right)$. Define $\bar{I}_{\max }(u)=\frac{1}{2} \int_{\mathbb{R}^{N}}\left(|\nabla u|^{2}+u^{2}\right) d z-\frac{1}{p} \int_{\mathbb{R}^{N}}|u|^{p} d z$ and $\bar{\theta}_{\max }=\inf _{u \in \overline{\mathbf{N}}_{\max }} \bar{I}_{\max }(u)$, where

$$
\left.\overline{\mathbf{N}}_{\max }=\left\{u \in H^{1}\left(\mathbb{R}^{N}\right) \backslash\{0\}|| \bar{I}_{\max }^{\prime}(u), u\right\rangle=0\right\} .
$$


Moreover, we have that

$$
\bar{\theta}_{\max }=\frac{p-2}{2 p} S_{p}^{\frac{p}{p-2}}>0 . \quad(\text { See Wang }[6, \text { Theorems } 4.12 \text { and 4.13].) }
$$

This paper is organized as follows. First of all, we study the argument of the Nehari manifold $\mathbf{M}_{\varepsilon, \lambda, \mu}$. Next, we prove that the existence of a positive solution $\left(u_{0}, v_{0}\right) \in \mathbf{M}_{\varepsilon, \lambda, \mu}$ of $\left(E_{\varepsilon, \lambda, \mu}\right)$. Finally, in Section 4, we show that the condition (A2) affects the number of positive solutions of $\left(E_{\varepsilon, \lambda, \mu}\right)$; that is, there are at least $k$ critical points $\left(u_{i}, v_{i}\right) \in \mathbf{M}_{\varepsilon, \lambda, \mu}$ of $J_{\varepsilon, \lambda, \mu}$ such that $J_{\varepsilon, \lambda, \mu}\left(u_{i}, v_{i}\right)=\beta_{\varepsilon, \lambda, \mu}^{i}((\mathrm{PS})$-value) for $1 \leq i \leq k$.

Theorem $1.1\left(E_{\varepsilon, \lambda, \mu}\right)$ has at least one positive solution $\left(u_{0}, v_{0}\right)$, that is, $\left(\bar{E}_{\varepsilon, \lambda, \mu}\right)$ admits at least one positive solution.

Theorem 1.2 There exist two positive numbers $\varepsilon_{0}$ and $\Lambda^{*}$ such that $\left(E_{\varepsilon, \lambda, \mu}\right)$ has at least $k$ positive solutions for any $0<\varepsilon<\varepsilon_{0}$ and $0<\lambda+\mu<\Lambda^{*}$, that is, $\left(\bar{E}_{\varepsilon, \lambda, \mu}\right)$ admits at least $k$ positive solutions.

\section{Preliminaries}

By studying the argument of Han [7, Lemma 2.1], we obtain the following lemma.

Lemma 2.1 Let $\Omega \subset \mathbb{R}^{N}$ (possibly unbounded) be a smooth domain. If $u_{n} \rightarrow u, v_{n} \rightarrow v$ weakly in $H_{0}^{1}(\Omega)$, and $u_{n} \rightarrow u, v_{n} \rightarrow v$ almost everywhere in $\Omega$, then

$$
\lim _{n \rightarrow \infty} \int_{\Omega}\left|u_{n}-u\right|^{\alpha}\left|v_{n}-v\right|^{\beta} d z=\lim _{n \rightarrow \infty} \int_{\Omega}\left|u_{n}\right|^{\alpha}\left|v_{n}\right|^{\beta} d z-\int_{\Omega}|u|^{\alpha}|v|^{\beta} d z
$$

Note that $J_{\varepsilon, \lambda, \mu}$ is not bounded from below in $H$. From the following lemma, we have that $J_{\varepsilon, \lambda, \mu}$ is bounded from below on $\mathbf{M}_{\varepsilon, \lambda, \mu}$.

Lemma 2.2 The energy functional $J_{\varepsilon, \lambda, \mu}$ is bounded from below on $\mathbf{M}_{\varepsilon, \lambda, \mu}$.

Proof For $(u, v) \in \mathbf{M}_{\varepsilon, \lambda, \mu}$, by (1.1), we obtain that

$$
J_{\varepsilon, \lambda, \mu}(u, v)=\left(\frac{1}{2}-\frac{1}{q}\right)\|(u, v)\|_{H}^{2}+\left(\frac{1}{q}-\frac{1}{p}\right) \int_{\mathbb{R}^{N}} f(\varepsilon z)|u|^{\alpha}|v|^{\beta} d z>0
$$

where $p=\alpha+\beta$. Hence, we have that $J_{\varepsilon, \lambda, \mu}$ is bounded from below on $\mathbf{M}_{\varepsilon, \lambda, \mu}$.

We define

$$
\theta_{\varepsilon, \lambda, \mu}=\inf _{(u, v) \in \mathbf{M}_{\varepsilon, \lambda, \mu}} J_{\varepsilon, \lambda, \mu}(u, v)
$$

Lemma 2.3 (i) There exist positive numbers $\sigma$ and $d_{0}$ such that $J_{\varepsilon, \lambda, \mu}(u, v) \geq d_{0}$ for $\|(u, v)\|_{H}=\sigma$;

(ii) There exists $(\bar{u}, \bar{v}) \in H \backslash\{(0,0)\}$ such that $\|(\bar{u}, \bar{v})\|_{H}>\sigma$ and $J_{\varepsilon, \lambda, \mu}(\bar{u}, \bar{v})<0$. 
Proof (i) By (1.2), the Hölder inequality $\left(p_{1}=\frac{p}{p-q}, p_{2}=\frac{p}{q}\right)$ and the Sobolev embedding theorem, we have that

$$
\begin{aligned}
J_{\varepsilon, \lambda, \mu}(u, v)= & \frac{1}{2}\|(u, v)\|_{H}^{2}-\frac{1}{p} \int_{\mathbb{R}^{N}} f(\varepsilon z)|u|^{\alpha}|v|^{\beta} d z \\
& -\frac{1}{q} \int_{\mathbb{R}^{N}}\left(\lambda g(\varepsilon z)|u|^{q}+\mu h(\varepsilon z)|v|^{q}\right) d z \\
\geq & \frac{1}{2}\|(u, v)\|_{H}^{2}-\frac{1}{p} S_{\alpha, \beta}^{-p / 2}\|(u, v)\|_{H}^{p} \\
& -\frac{1}{q} \operatorname{Max} S_{p}^{-\frac{q}{2}}(\lambda+\mu)\|(u, v)\|_{H}^{q},
\end{aligned}
$$

where $p=\alpha+\beta$ and $\operatorname{Max}=\max \left\{\|g\|_{m},\|h\|_{m}\right\}$. Hence, there exist positive $\sigma$ and $d_{0}$ such that $J_{\varepsilon, \lambda, \mu}(u, v) \geq d_{0}$ for $\|(u, v)\|_{H}=\sigma$.

(ii) For any $(u, v) \in H \backslash\{(0,0)\}$, since

$$
\begin{aligned}
J_{\varepsilon, \lambda, \mu}(t u, t v)= & \frac{t^{2}}{2}\|(u, v)\|_{H}^{2}-\frac{t^{p}}{p} \int_{\mathbb{R}^{N}} f(\varepsilon z)|u|^{\alpha}|v|^{\beta} d z \\
& -\frac{t^{q}}{q} \int_{\mathbb{R}^{N}}\left(\lambda g(\varepsilon z)|u|^{q}+\mu h(\varepsilon z)|v|^{q}\right) d z,
\end{aligned}
$$

then $\lim _{t \rightarrow \infty} J_{\varepsilon, \lambda, \mu}(t u, t v)=-\infty$. Fix some $(u, v) \in H \backslash\{(0,0)\}$, there exists $\bar{t}>0$ such that $\|(\bar{t} u, \bar{t} v)\|_{H}>\sigma$ and $J_{\varepsilon, \lambda, \mu}(\bar{t} u, \bar{t} v)<0$. Let $(\bar{u}, \bar{v})=(\bar{t} u, \bar{t} v)$.

Define

$$
\psi(u, v)=\left\langle J_{\varepsilon, \lambda, \mu}^{\prime}(u, v),(u, v)\right\rangle .
$$

Then for $(u, v) \in \mathbf{M}_{\varepsilon, \lambda, \mu}$, we obtain that

$$
\begin{aligned}
\left\langle\psi^{\prime}(u, v),(u, v)\right\rangle= & 2\|(u, v)\|_{H}^{2}-p \int_{\mathbb{R}^{N}} f(\varepsilon z)|u|^{\alpha}|v|^{\beta} d z \\
& -q \int_{\mathbb{R}^{N}}\left(\lambda g(\varepsilon z)|u|^{q}+\mu h(\varepsilon z)|v|^{q}\right) d z \\
= & (p-q) \int_{\mathbb{R}^{N}}\left(\lambda g(\varepsilon z)|u|^{q}+\mu h(\varepsilon z)|v|^{q}\right) d z-(p-2)\|(u, v)\|_{H}^{2} \\
= & (2-q)\|(u, v)\|_{H}^{2}+(q-p) \int_{\mathbb{R}^{N}} f(\varepsilon z)|u|^{\alpha}|v|^{\beta} d z<0 .
\end{aligned}
$$

Lemma 2.4 For each $(u, v) \in H \backslash\{(0,0)\}$, there exists a unique positive number $t_{u, v}$ such that $\left(t_{u, v} u, t_{u, v} v\right) \in \mathbf{M}_{\varepsilon, \lambda, \mu}$ and $J_{\varepsilon, \lambda, \mu}\left(t_{u, v} u, t_{u, v} v\right)=\sup _{t \geq 0} J_{\varepsilon, \lambda, \mu}(t u, t v)$.

Proof Fixed $(u, v) \in H \backslash\{(0,0)\}$, we consider

$$
\begin{aligned}
R(t) & =J_{\varepsilon, \lambda, \mu}(t u, t v) \\
& =\frac{t^{2}}{2}\|(u, v)\|_{H}^{2}-\frac{t^{p}}{p} \int_{\mathbb{R}^{N}} f(\varepsilon z)|u|^{\alpha}|v|^{\beta} d z-\frac{t^{q}}{q} \int_{\mathbb{R}^{N}}\left(\lambda g(\varepsilon z)|u|^{q}+\mu h(\varepsilon z)|v|^{q}\right) d z .
\end{aligned}
$$


Since $R(0)=0, \lim _{t \rightarrow \infty} R(t)=-\infty$, by Lemma $2.3(\mathrm{i})$, then $\sup _{t \geq 0} R(t)$ is achieved at some $t_{u, v}>0$. Moreover, we have that $R^{\prime}\left(t_{u, v}\right)=0$, that is, $\left(t_{u, v} u, t_{u, v} v\right) \in \mathbf{M}_{\varepsilon, \lambda, \mu}$. Next, we claim that $t_{u, v}$ is a unique positive number such that $R^{\prime}\left(t_{u, v}\right)=0$. Consider

$$
r(t)=\|(u, v)\|_{H}^{2}-t^{p-2} \int_{\mathbb{R}^{N}} f(\varepsilon z)|u|^{\alpha}|v|^{\beta} d z-t^{q-2} \int_{\mathbb{R}^{N}}\left(\lambda g(\varepsilon z)|u|^{q}+\mu h(\varepsilon z)|v|^{q}\right) d z,
$$

then $R^{\prime}(t)=\operatorname{tr}(t)$. Since $r(0)=\|(u, v)\|_{H}^{2}>0$,

$$
\begin{aligned}
r^{\prime}(t)= & -(p-2) t^{p-3} \int_{\mathbb{R}^{N}} f(\varepsilon z)|u|^{\alpha}|v|^{\beta} d z \\
& -(q-2) t^{q-3} \int_{\mathbb{R}^{N}}\left(\lambda g(\varepsilon z)|u|^{q}+\mu h(\varepsilon z)|v|^{q}\right) d z<0,
\end{aligned}
$$

there exists a unique positive number $\bar{t}_{u, v}$ such that $r\left(\bar{t}_{u, v}\right)=0$. It follows that $R^{\prime}\left(\bar{t}_{u, v}\right)=0$. Hence, $\bar{t}_{u, v}=t_{u, v}$.

Remark 2.5 By Lemma 2.3(i) and Lemma 2.4, then $\theta_{\varepsilon, \lambda, \mu} \geq d_{0}>0$ for some constant $d_{0}$.

Lemma 2.6 Let $\left(u_{0}, v_{0}\right) \in \mathbf{M}_{\varepsilon, \lambda, \mu}$ satisfy

$$
J_{\varepsilon, \lambda, \mu}\left(u_{0}, v_{0}\right)=\min _{(u, v) \in \mathbf{M}_{\varepsilon, \lambda, \mu}} J_{\varepsilon, \lambda, \mu}(u, v)=\theta_{\varepsilon, \lambda, \mu}
$$

then $\left(u_{0}, v_{0}\right)$ is a solution of $\left(E_{\varepsilon, \lambda, \mu}\right)$.

Proof By (2.2), $\left\langle\psi^{\prime}(u, v),(u, v)\right\rangle<0$ for $(u, v) \in \mathbf{M}_{\varepsilon, \lambda, \mu}$. Since $J_{\varepsilon, \lambda, \mu}\left(u_{0}, v_{0}\right)=$ $\min _{(u, v) \in \mathbf{M}_{\varepsilon, \lambda, \mu}} J_{\varepsilon, \lambda, \mu}(u, v)$, by the Lagrange multiplier theorem, there is $\tau \in \mathbb{R}$ such that $J_{\varepsilon, \lambda, \mu}^{\prime}\left(u_{0}, v_{0}\right)=\tau \psi^{\prime}\left(u_{0}, v_{0}\right)$ in $H^{-1}$. Then we have

$$
0=\left\langle J_{\varepsilon, \lambda, \mu}^{\prime}\left(u_{0}, v_{0}\right),\left(u_{0}, v_{0}\right)\right\rangle=\tau\left\langle\psi^{\prime}\left(u_{0}, v_{0}\right),\left(u_{0}, v_{0}\right)\right\rangle \text {. }
$$

It follows that $\tau=0$ and $J_{\varepsilon, \lambda, \mu}^{\prime}\left(u_{0}, v_{0}\right)=0$ in $H^{-1}$. Therefore, $\left(u_{0}, v_{0}\right)$ is a nontrivial solution of $\left(E_{\varepsilon, \lambda, \mu}\right)$ and $J_{\varepsilon, \lambda, \mu}\left(u_{0}, v_{0}\right)=\theta_{\varepsilon, \lambda, \mu}$.

\section{3 (PS $_{\gamma}$-condition in $H$ for $J_{\varepsilon, \lambda, \mu}$}

First of all, we define the Palais-Smale (denoted by (PS)) sequence and (PS)-condition in $H$ for some functional $J$.

Definition 3.1 (i) For $\gamma \in \mathbb{R}$, a sequence $\left\{\left(u_{n}, v_{n}\right)\right\}$ is a (PS) $)_{\gamma}$-sequence in $H$ for $J$ if $J\left(u_{n}, v_{n}\right)=\gamma+o_{n}(1)$ and $J^{\prime}\left(u_{n}, v_{n}\right)=o_{n}(1)$ strongly in $H^{-1}$ as $n \rightarrow \infty$, where $H^{-1}$ is the dual space of $H$;

(ii) $J$ satisfies the $(\mathrm{PS})_{\gamma}$-condition in $H$ if every $(\mathrm{PS})_{\gamma}$-sequence in $H$ for $J$ contains a convergent subsequence.

Applying Ekeland's variational principle and using the same argument as in Cao-Zhou [8] or Tarantello [9], we have the following lemma.

Lemma 3.2 (i) There exists a $(P S)_{\theta_{\varepsilon, \lambda, \mu}}$-sequence $\left\{\left(u_{n}, v_{n}\right)\right\}$ in $\mathbf{M}_{\varepsilon, \lambda, \mu}$ for $J_{\varepsilon, \lambda, \mu}$. 
In order to prove the existence of positive solutions, we want to prove that $J_{\varepsilon, \lambda, \mu}$ satisfies the $(\mathrm{PS})_{\gamma}$-condition in $H$ for $\gamma \in\left(0, \frac{p-2}{2 p} \frac{\left(S_{\alpha, \beta}\right)^{p /(p-2)}}{\left(f_{\infty}\right)^{2 /(p-2)}}\right)$.

Lemma $3.3 J_{\varepsilon, \lambda, \mu}$ satisfies the $(P S)_{\gamma}$-condition in $H$ for $\gamma \in\left(0, \frac{p-2}{2 p} \frac{\left(S_{\alpha, \beta}\right)^{p /(p-2)}}{\left(f_{\infty}\right)^{2 /(p-2)}}\right)$.

Proof Let $\left\{\left(u_{n}, v_{n}\right)\right\}$ be a (PS) $)_{\gamma}$-sequence in $H$ for $J_{\varepsilon, \lambda, \mu}$ such that $J_{\varepsilon, \lambda, \mu}\left(u_{n}, v_{n}\right)=\gamma+o_{n}(1)$ and $J_{\varepsilon, \lambda, \mu}^{\prime}\left(u_{n}, v_{n}\right)=o_{n}(1)$ in $H^{-1}$. Then

$$
\begin{aligned}
\gamma+c_{n}+\frac{d_{n}\left\|\left(u_{n}, v_{n}\right)\right\|_{H}}{q} & \geq J_{\varepsilon, \lambda, \mu}\left(u_{n}, v_{n}\right)-\frac{1}{q}\left\langle J_{\varepsilon, \lambda, \mu}^{\prime}\left(u_{n}, v_{n}\right),\left(u_{n}, v_{n}\right)\right\rangle \\
& =\left(\frac{1}{2}-\frac{1}{q}\right)\left\|\left(u_{n}, v_{n}\right)\right\|_{H}^{2}+\left(\frac{1}{q}-\frac{1}{p}\right) \int_{\mathbb{R}^{N}} f(\varepsilon z)\left|u_{n}\right|^{\alpha}\left|v_{n}\right|^{\beta} d z \\
& \geq \frac{q-2}{2 q}\left\|\left(u_{n}, v_{n}\right)\right\|_{H}^{2}
\end{aligned}
$$

where $c_{n}=o_{n}(1), d_{n}=o_{n}(1)$ as $n \rightarrow \infty$. It follows that $\left\{\left(u_{n}, v_{n}\right)\right\}$ is bounded in $H$. Hence, there exist a subsequence $\left\{\left(u_{n}, v_{n}\right)\right\}$ and $(u, v) \in H$ such that

$$
\begin{array}{ll}
u_{n} \rightarrow u, & v_{n} \rightarrow v \quad \text { weakly in } H^{1}\left(\mathbb{R}^{N}\right) ; \\
u_{n} \rightarrow u, & v_{n} \rightarrow v \quad \text { strongly in } L_{\text {loc }}^{s}\left(\mathbb{R}^{N}\right) \text { for any } 1 \leq s<2^{*} ; \\
u_{n} \rightarrow u, & v_{n} \rightarrow v \quad \text { a.e. in } \mathbb{R}^{N} .
\end{array}
$$

Moreover, we have that $J_{\varepsilon, \lambda, \mu}^{\prime}(u, v)=0$ in $H^{-1}$. We use the Brézis-Lieb lemma to obtain (3.1) and (3.2) as follows:

$$
\begin{aligned}
& \int_{\mathbb{R}^{N}} g(\varepsilon z)\left|u_{n}-u\right|^{q} d z=\int_{\mathbb{R}^{N}} g(\varepsilon z)\left|u_{n}\right|^{q} d z-\int_{\mathbb{R}^{N}} g(\varepsilon z)|u|^{q} d z+o_{n}(1) ; \\
& \int_{\mathbb{R}^{N}} h(\varepsilon z)\left|v_{n}-v\right|^{q} d z=\int_{\mathbb{R}^{N}} h(\varepsilon z)\left|v_{n}\right|^{q} d z-\int_{\mathbb{R}^{N}} h(\varepsilon z)|v|^{q} d z+o_{n}(1) .
\end{aligned}
$$

Next, we claim that

$$
\int_{\mathbb{R}^{N}} g(\varepsilon z)\left|u_{n}-u\right|^{q} d z \rightarrow 0 \quad \text { as } n \rightarrow \infty
$$

and

$$
\int_{\mathbb{R}^{N}} h(\varepsilon z)\left|v_{n}-v\right|^{q} d z \rightarrow 0 \quad \text { as } n \rightarrow \infty
$$

Since $g \in L^{m}\left(\mathbb{R}^{N}\right)$, where $m=p /(p-q)$, then for any $\sigma>0$, there exists $r>0$ such that $\int_{\left[B_{r}^{N}(0)\right]^{c}} g(\varepsilon z)^{\frac{p}{p-q}} d z<\sigma$. By the Hölder inequality and the Sobolev embedding theorem, we get

$$
\begin{aligned}
& \left|\int_{\mathbb{R}^{N}} g(\varepsilon z)\right| u_{n}-\left.u\right|^{q} d z \mid \\
& \quad \leq \int_{B_{r}^{N}(0)} g(\varepsilon z)\left|u_{n}-u\right|^{q} d z
\end{aligned}
$$




$$
\begin{aligned}
& +\int_{\left.\left[B_{r}^{N}(0)\right]\right]^{c}} g(\varepsilon z)\left|u_{n}-u\right|^{q} d z \\
\leq & \|g\|_{m}\left(\int_{B_{r}^{N}(0)}\left|u_{n}-u\right|^{p} d z\right)^{q / p} \\
& +S_{p}^{-\frac{q}{2}}\left(\int_{\left[B_{r}^{N}(0)\right]^{c}} g(\varepsilon z)^{\frac{p}{p-q}} d z\right)^{\frac{p-q}{p}}\left(\int_{\mathbb{R}^{N}}\left|\nabla\left(u_{n}-u\right)\right|^{2}+\left|u_{n}-u\right|^{2} d z\right)^{q / 2} \\
\leq & C^{\prime} \sigma+o_{n}(1) \quad\left(\because\left\{u_{n}\right\} \text { is bounded in } H^{1}\left(\mathbb{R}^{N}\right) \text { and } u_{n} \rightarrow u \text { in } L_{\mathrm{loc}}^{p}\left(\mathbb{R}^{N}\right)\right) .
\end{aligned}
$$

Similarly, $\int_{\mathbb{R}^{N}} h(\varepsilon z)\left|v_{n}-v\right|^{q} d z \rightarrow 0$ as $n \rightarrow \infty$. By (A1) and $u_{n} \rightarrow u, v_{n} \rightarrow v$ strongly in $L_{\mathrm{loc}}^{p}\left(\mathbb{R}^{N}\right)$, we have that

$$
\int_{\mathbb{R}^{N}} f(\varepsilon z)\left|u_{n}-u\right|^{\alpha}\left|v_{n}-v\right|^{\beta} d z=\int_{\mathbb{R}^{N}} f_{\infty}\left|u_{n}-u\right|^{\alpha}\left|v_{n}-v\right|^{\beta} d z=o_{n}(1)
$$

Let $p_{n}=\left(u_{n}-u, v_{n}-v\right)$. By (3.1)-(3.4) and Lemma 2.1, we deduce that

$$
\begin{aligned}
\left\|p_{n}\right\|_{H}^{2}= & \left(\left\|u_{n}\right\|_{H}^{2}+\left\|v_{n}\right\|_{H}^{2}\right)-\left(\|u\|_{H}^{2}+\|v\|_{H}^{2}\right)+o_{n}(1) \\
= & \int_{\mathbb{R}^{N}} f(\varepsilon z)\left|u_{n}\right|^{\alpha}\left|v_{n}\right|^{\beta} d z+\int_{\mathbb{R}^{N}}\left(\lambda g(\varepsilon z)\left|u_{n}\right|^{q}+\mu h(\varepsilon z)\left|v_{n}\right|^{q}\right) d z \\
& -\int_{\mathbb{R}^{N}} f(\varepsilon z)|u|^{\alpha}|v|^{\beta} d z-\int_{\mathbb{R}^{N}}\left(\lambda g(\varepsilon z)|u|^{q}+\mu h(\varepsilon z)|v|^{q}\right) d z+o_{n}(1) \\
= & \int_{\mathbb{R}^{N}} f(\varepsilon z)\left|u_{n}-u\right|^{\alpha}\left|v_{n}-v\right|^{\beta} d z+o_{n}(1)
\end{aligned}
$$

and

$$
\frac{1}{2}\left\|p_{n}\right\|_{H}^{2}-\frac{1}{\alpha+\beta} \int_{\mathbb{R}^{N}} f(\varepsilon z)\left|u_{n}-u\right|^{\alpha}\left|v_{n}-v\right|^{\beta} d z=\gamma-J_{\varepsilon, \lambda, \mu}(u, v)+o_{n}(1) .
$$

We may assume that

$$
\left\|p_{n}\right\|_{H}^{2} \rightarrow l \text { and } \int_{\mathbb{R}^{N}} f(\varepsilon z)\left|u_{n}-u\right|^{\alpha}\left|v_{n}-v\right|^{\beta} d z \rightarrow l \quad \text { as } n \rightarrow \infty
$$

Recall that

$$
S_{\alpha, \beta}=\inf _{u, v \in H^{1}\left(\mathbb{R}^{N}\right) \backslash\{(0)\}} \frac{\|(u, v)\|_{H}^{2}}{\left(\int_{\mathbb{R}^{N}}|u|^{\alpha}|v|^{\beta} d z\right)^{2 / p}}, \quad \text { where } p=\alpha+\beta .
$$

If $l>0$, by (3.5), then

$$
\begin{aligned}
S_{\alpha, \beta} l^{\frac{2}{p}} & =S_{\alpha, \beta}\left(\int_{\mathbb{R}^{N}} f(\varepsilon z)\left|u_{n}-u\right|^{\alpha}\left|v_{n}-v\right|^{\beta} d z\right)^{2 / p}+o_{n}(1) \\
& =S_{\alpha, \beta}\left(\int_{\mathbb{R}^{N}} f_{\infty}\left|u_{n}-u\right|^{\alpha}\left|v_{n}-v\right|^{\beta} d z\right)^{2 / p}+o_{n}(1) \\
& \leq\left(f_{\infty}\right)^{2 / p}\left\|p_{n}\right\|_{H}^{2}+o_{n}(1)=\left(f_{\infty}\right)^{2 / p} l .
\end{aligned}
$$


This implies $l \geq\left(S_{\alpha, \beta}\right)^{p /(p-2)} /\left(f_{\infty}\right)^{2 /(p-2)}$. By (3.6) and (3.7), we obtain that

$$
\gamma=\left(\frac{1}{2}-\frac{1}{p}\right) l+J_{\varepsilon, \lambda, \mu}(u, v) \geq \frac{p-2}{2 p} \frac{\left(S_{\alpha, \beta}\right)^{p /(p-2)}}{\left(f_{\infty}\right)^{2 /(p-2)}},
$$

which is a contradiction. Hence, $l=0$, that is, $\left(u_{n}, v_{n}\right) \rightarrow(u, v)$ strongly in $H$.

\section{Existence of $k$ solutions}

Let $w \in H^{1}\left(\mathbb{R}^{N}\right)$ be the unique, radially symmetric and positive ground state solution of equation (E0) in $\mathbb{R}^{N}$. Recall the facts (or see Bahri-Li [10], Bahri-Lions [11], Gidas-NiNirenberg [12] and Kwong [13]):

(i) $w \in L^{\infty}\left(\mathbb{R}^{N}\right) \cap C_{\text {loc }}^{2, \theta}\left(\mathbb{R}^{N}\right)$ for some $0<\theta<1$ and $\lim _{|z| \rightarrow \infty} w(z)=0$;

(ii) for any $\varepsilon>0$, there exist positive numbers $C_{1}, C_{2}^{\varepsilon}$ and $C_{3}^{\varepsilon}$ such that for all $z \in \mathbb{R}^{N}$

$$
C_{2}^{\varepsilon} \exp (-(1+\varepsilon)|z|) \leq w(z) \leq C_{1} \exp (-|z|)
$$

and

$$
|\nabla w(z)| \leq C_{3}^{\varepsilon} \exp (-(1-\varepsilon)|z|)
$$

By Lien-Tzeng-Wang [14], then

$$
S_{p}=\frac{\int_{\mathbb{R}^{N}}\left(|\nabla w|^{2}+w^{2}\right) d z}{\left(\int_{\mathbb{R}^{N}} w^{p} d z\right)^{2 / p}}
$$

For $1 \leq i \leq k$, we define

$$
w_{\varepsilon}^{i}(z)=w\left(z-\frac{a^{i}}{\varepsilon}\right), \quad \text { where } f\left(a^{i}\right)=\max _{z \in \mathbb{R}^{N}} f(z)=1 .
$$

Clearly, $w_{\varepsilon}^{i}(z) \in H^{1}\left(\mathbb{R}^{N}\right)$.

First of all, we want to prove that

$$
\lim _{\varepsilon \rightarrow 0^{+}} \sup _{t \geq 0} J_{\varepsilon, \lambda, \mu}\left(t \sqrt{\alpha} w_{\varepsilon}^{i}, t \sqrt{\beta} w_{\varepsilon}^{i}\right) \leq \frac{p-2}{2 p}\left(S_{\alpha, \beta}\right)^{p /(p-2)} \quad \text { uniformly in } i \text {. }
$$

Lemma 4.1 For $\lambda>0$ and $\mu>0$, then

$$
\lim _{\varepsilon \rightarrow 0^{+}} \sup _{t \geq 0} J_{\varepsilon, \lambda, \mu}\left(t \sqrt{\alpha} w_{\varepsilon}^{i}, t \sqrt{\beta} w_{\varepsilon}^{i}\right) \leq \frac{p-2}{2 p}\left(S_{\alpha, \beta}\right)^{p /(p-2)} \quad \text { uniformly in } i .
$$

Moreover, we have that

$$
0<\theta_{\varepsilon, \lambda, \mu} \leq \frac{p-2}{2 p}\left(S_{\alpha, \beta}\right)^{p /(p-2)}
$$

Proof Part I: Since $J_{\varepsilon, \lambda, \mu}$ is continuous in $H, J_{\varepsilon, \lambda, \mu}(0,0)=0$, and $\left\{\left(\sqrt{\alpha} w_{\varepsilon}^{i}, \sqrt{\beta} w_{\varepsilon}^{i}\right)\right\}$ is uniformly bounded in $H$ for any $\varepsilon>0$ and $1 \leq i \leq k$, then there exists $t_{0}>0$ such that for $0 \leq t<t_{0}$ and any $\varepsilon>0$,

$$
J_{\varepsilon, \lambda, \mu}\left(t \sqrt{\alpha} w_{\varepsilon}^{i}, t \sqrt{\beta} w_{\varepsilon}^{i}\right)<\frac{p-2}{2 p}\left(S_{\alpha, \beta}\right)^{p /(p-2)} \quad \text { uniformly in } i .
$$


From (A1), we have that $\inf _{z \in \mathbb{R}^{N}} f(z)>0$. Then

$$
\begin{aligned}
J_{\varepsilon, \lambda, \mu}\left(t \sqrt{\alpha} w_{\varepsilon}^{i}, t \sqrt{\beta} w_{\varepsilon}^{i}\right) \leq & \frac{t^{2}}{2}\|(\sqrt{\alpha} w, \sqrt{\beta} w)\|_{H}^{2} \\
& -\frac{t^{\alpha+\beta}}{\alpha+\beta}\left(\inf _{z \in \mathbb{R}^{N}} f(z)\right) \int_{\mathbb{R}^{N}}|\sqrt{\alpha} w|^{\alpha}|\sqrt{\beta} w|^{\beta} d z \\
\rightarrow & -\infty \quad \text { as } t \rightarrow \infty .
\end{aligned}
$$

It follows that there exists $t_{1}>0$ such that for $t>t_{1}$ and any $\varepsilon>0$,

$$
J_{\varepsilon, \lambda, \mu}\left(t \sqrt{\alpha} w_{\varepsilon}^{i}, t \sqrt{\beta} w_{\varepsilon}^{i}\right)<\frac{p-2}{2 p}\left(S_{\alpha, \beta}\right)^{p /(p-2)} \quad \text { uniformly in } i .
$$

From now on, we only need to show that

$$
\lim _{\varepsilon \rightarrow 0^{+}} \sup _{t_{0} \leq t \leq t_{1}} J_{\varepsilon, \lambda, \mu}\left(t w_{\varepsilon}^{i}\right) \leq \frac{p-2}{2 p}\left(S_{\alpha, \beta}\right)^{p /(p-2)} \quad \text { uniformly in } i .
$$

Since

$$
\sup _{t \geq 0}\left(\frac{t^{2}}{2} a-\frac{t^{\alpha+\beta}}{\alpha+\beta} b\right)=\frac{\alpha+\beta-2}{2(\alpha+\beta)}\left(\frac{a}{b^{\frac{2}{\alpha+\beta}}}\right)^{\frac{\alpha+\beta}{\alpha+\beta-2}}, \quad \text { where } a, b>0
$$

and by (4.1), then

$$
\begin{gathered}
\sup _{t \geq 0}\left\{\frac{t^{2}}{2}\left\|\left(\sqrt{\alpha} w_{\varepsilon}^{i}, \sqrt{\beta} w_{\varepsilon}^{i}\right)\right\|_{H}^{2}-\frac{t^{\alpha+\beta}}{\alpha+\beta} \int_{\mathbb{R}^{N}}\left|\sqrt{\alpha} w_{\varepsilon}^{i}\right|^{\alpha}\left|\sqrt{\alpha} w_{\varepsilon}^{i}\right|^{\beta} d z\right\} \\
=\frac{p-2}{2 p}\left[\frac{(\alpha+\beta) \int_{\mathbb{R}^{N}}\left(|\nabla w|^{2}+w^{2}\right) d z}{\left(\alpha^{\frac{\alpha}{2}} \beta^{\frac{\beta}{2}} \int_{\mathbb{R}^{N}} w^{p} d z\right)^{2 / p}}\right]^{\frac{p}{p-2}}=\frac{p-2}{2 p}\left(S_{\alpha, \beta}\right)^{p /(p-2)}
\end{gathered}
$$

For $t_{0} \leq t \leq t_{1}$, by (4.2), we have that

$$
\begin{aligned}
J_{\varepsilon, \lambda, \mu}\left(t \sqrt{\alpha} w_{\varepsilon}^{i}, t \sqrt{\beta} w_{\varepsilon}^{i}\right)= & \frac{t^{2}}{2}\left\|\left(\sqrt{\alpha} w_{\varepsilon}^{i}, \sqrt{\beta} w_{\varepsilon}^{i}\right)\right\|_{H}^{2}-\frac{t^{\alpha+\beta}}{\alpha+\beta} \int_{\mathbb{R}^{N}} f(\varepsilon z)\left|\sqrt{\alpha} w_{\varepsilon}^{i}\right|^{\alpha}\left|\sqrt{\beta} w_{\varepsilon}^{i}\right|^{\beta} d z \\
& -\frac{t^{q}}{q} \int_{\mathbb{R}^{N}}\left(\lambda g(\varepsilon z)\left|\sqrt{\alpha} w_{\varepsilon}^{i}\right|^{q}+\mu h(\varepsilon z)\left|\sqrt{\beta} w_{\varepsilon}^{i}\right|^{q}\right) d z \\
\leq & \frac{p-2}{2 p}\left(S_{\alpha, \beta}\right)^{p /(p-2)} \\
& +\frac{t_{1}^{p}}{p} \int_{\mathbb{R}^{N}}(1-f(\varepsilon z))\left|\sqrt{\alpha} w_{\varepsilon}^{i}\right|^{\alpha}\left|\sqrt{\alpha} w_{\varepsilon}^{i}\right|^{\beta} d z .
\end{aligned}
$$

Since

$$
\begin{aligned}
& \int_{\mathbb{R}^{N}}(1-f(\varepsilon z))\left|\sqrt{\alpha} w_{\varepsilon}^{i}\right|^{\alpha}\left|\sqrt{\beta} w_{\varepsilon}^{i}\right|^{\beta} d z \\
& \quad=\alpha^{\frac{\alpha}{2}} \beta^{\frac{\beta}{2}} \int_{\mathbb{R}^{N}}\left(1-f\left(\varepsilon z+a^{i}\right)\right) w^{p} d z=o(1) \quad \text { as } \varepsilon \rightarrow 0^{+} \text {uniformly in } i,
\end{aligned}
$$


then

$$
\lim _{\varepsilon \rightarrow 0^{+}} \sup _{t_{0} \leq t \leq t_{1}} J_{\varepsilon, \lambda, \mu}\left(t \sqrt{\alpha} w_{\varepsilon}^{i}, t \sqrt{\beta} w_{\varepsilon}^{i}\right) \leq \frac{p-2}{2 p}\left(S_{\alpha, \beta}\right)^{p /(p-2)},
$$

that is, for $\lambda>0$ and $\mu>0$,

$$
\lim _{\varepsilon \rightarrow 0^{+}} \sup _{t \geq 0} J_{\varepsilon, \lambda, \mu}\left(t \sqrt{\alpha} w_{\varepsilon}^{i}, t \sqrt{\beta} w_{\varepsilon}^{i}\right) \leq \frac{p-2}{2 p}\left(S_{\alpha, \beta}\right)^{p /(p-2)} \quad \text { uniformly in } i .
$$

Part II: By Lemma 2.4, there is a number $t_{\varepsilon}^{i}>0$ such that $\left(t_{\varepsilon}^{i} \sqrt{\alpha} w_{\varepsilon}^{i}, t_{\varepsilon}^{i} \sqrt{\beta} w_{\varepsilon}^{i}\right) \in \mathbf{M}_{\varepsilon, \lambda, \mu}$, where $1 \leq i \leq k$. Hence, from the result of Part $\mathrm{I}$, we have that for $\lambda>0$ and $\mu>0$,

$$
0<\theta_{\varepsilon, \lambda, \mu} \leq \lim _{\varepsilon \rightarrow 0^{+}} \sup _{t \geq 0} J_{\varepsilon, \lambda, \mu}\left(t \sqrt{\alpha} w_{\varepsilon}^{i}, t \sqrt{\beta} w_{\varepsilon}^{i}\right) \leq \frac{p-2}{2 p}\left(S_{\alpha, \beta}\right)^{p /(p-2)} .
$$

Proof of Theorem 1.1 By Lemma 3.2, there exists a (PS) $)_{\varepsilon, \lambda, \mu}$-sequence $\left\{\left(u_{n}, v_{n}\right)\right\}$ in $\mathbf{M}_{\varepsilon, \lambda, \mu}$ for $J_{\varepsilon, \lambda, \mu}$. Since $0<\theta_{\varepsilon, \lambda, \mu} \leq \frac{p-2}{2 p}\left(S_{\alpha, \beta}\right)^{p /(p-2)}<\frac{p-2}{2 p} \frac{\left(S_{\alpha, \beta}\right)^{p /(p-2)}}{\left(f_{\infty}\right)^{2 /(p-2)}}$ for $\lambda>0$ and $\mu>0$, by Lemma 3.3, there exist a subsequence $\left\{\left(u_{n}, v_{n}\right)\right\}$ and $\left(u_{0}, v_{0}\right) \in H$ such that $\left(u_{n}, v_{n}\right) \rightarrow\left(u_{0}, v_{0}\right)$ strongly in $H$. It is easy to check that $\left(u_{0}, v_{0}\right)$ is a nontrivial solution of $\left(E_{\varepsilon, \lambda, \mu}\right)$ and $J_{\varepsilon, \lambda, \mu}\left(u_{0}, v_{0}\right)=$ $\theta_{\varepsilon, \lambda, \mu}$. Since $J_{\varepsilon, \lambda, \mu}\left(u_{0}, v_{0}\right)=J_{\lambda, \mu}\left(\left|u_{0}\right|,\left|v_{0}\right|\right)$ and $\left(\left|u_{0}\right|,\left|v_{0}\right|\right) \in \mathbf{M}_{\varepsilon, \lambda, \mu}$, by Lemma 2.6, we may assume that $u_{0} \geq 0, v_{0} \geq 0$. Applying the maximum principle, $u_{0}>0$ and $v_{0}>0$ in $\Omega$.

Choosing $0<\rho_{0}<1$ such that

$$
\overline{B_{\rho_{0}}^{N}\left(a^{i}\right)} \cap \overline{B_{\rho_{0}}^{N}\left(a^{j}\right)}=\varnothing \quad \text { for } i \neq j \text { and } 1 \leq i, j \leq k,
$$

where $\overline{B_{\rho_{0}}^{N}\left(a^{i}\right)}=\left\{z \in \mathbb{R}^{N}|| z-a^{i} \mid \leq \rho_{0}\right\}$ and $f\left(a^{i}\right)=\max _{z \in \mathbb{R}^{N}} f(z)=1$, define $\mathbf{K}=\left\{a^{i} \mid 1 \leq i \leq k\right\}$ and $\mathbf{K}_{\rho_{0} / 2}=\bigcup_{i=1}^{k} \overline{B_{\rho_{0} / 2}^{N}\left(a^{i}\right)}$. Suppose $\bigcup_{i=1}^{k} \overline{B_{\rho_{0}}^{N}\left(a^{i}\right)} \subset B_{r_{0}}^{N}(0)$ for some $r_{0}>0$. Let $Q_{\varepsilon}$ be given by

$$
Q_{\varepsilon}(u, v)=\frac{\int_{\mathbb{R}^{N}} \chi(\varepsilon z)|u|^{\alpha}|v|^{\beta} d z}{\int_{\mathbb{R}^{N}}|u|^{\alpha}|v|^{\beta} d z}
$$

where $\chi: \mathbb{R}^{N} \rightarrow \mathbb{R}^{N}, \chi(z)=z$ for $|z| \leq r_{0}$ and $\chi(z)=r_{0} z /|z|$ for $|z|>r_{0}$.

For each $1 \leq i \leq k$, we define

$$
\begin{aligned}
& O_{\varepsilon, \lambda, \mu}^{i}=\left\{(u, v) \in \mathbf{M}_{\varepsilon, \lambda, \mu}|| Q_{\varepsilon}(u, v)-a^{i} \mid<\rho_{0}\right\}, \\
& \partial O_{\varepsilon, \lambda, \mu}^{i}=\left\{(u, v) \in \mathbf{M}_{\varepsilon, \lambda, \mu}|| Q_{\varepsilon}(u, v)-a^{i} \mid=\rho_{0}\right\}, \\
& \beta_{\varepsilon, \lambda, \mu}^{i}=\inf _{(u, v) \in O_{\varepsilon, \lambda, \mu}^{i}} J_{\varepsilon, \lambda, \mu}(u, v) \text { and } \widetilde{\beta}_{\varepsilon, \lambda, \mu}^{i}=\inf _{(u, v) \in \partial O_{\varepsilon, \lambda, \mu}^{i}} J_{\varepsilon, \lambda, \mu}(u, v) .
\end{aligned}
$$

By Lemma 2.4, there exists $t_{\varepsilon}^{i}>0$ such that $\left(t_{\varepsilon}^{i} \sqrt{\alpha} w_{\varepsilon}^{i}, t_{\varepsilon}^{i} \sqrt{\beta} w_{\varepsilon}^{i}\right) \in \mathbf{M}_{\varepsilon, \lambda, \mu}$ for each $1 \leq i \leq k$. Then we have the following result.

Lemma 4.2 There exists $\varepsilon_{1}>0$ such that if $\varepsilon \in\left(0, \varepsilon_{1}\right)$, then $Q_{\varepsilon}\left(t_{\varepsilon}^{i} \sqrt{\alpha} w_{\varepsilon}^{i}, t_{\varepsilon}^{i} \sqrt{\beta} w_{\varepsilon}^{i}\right) \in \mathbf{K}_{\rho_{0} / 2}$ for each $1 \leq i \leq k$. 
Proof Since

$$
\begin{aligned}
Q_{\varepsilon}\left(t_{\varepsilon}^{i} \sqrt{\alpha} w_{\varepsilon}^{i}, t_{\varepsilon}^{i} \sqrt{\beta} w_{\varepsilon}^{i}\right) & =\frac{\int_{\mathbb{R}^{N}} \chi(\varepsilon z)\left|w\left(z-\frac{a^{i}}{\varepsilon}\right)\right|^{p} d z}{\int_{\mathbb{R}^{N}}\left|w\left(z-\frac{a^{i}}{\varepsilon}\right)\right|^{p} d z} \\
& =\frac{\int_{\mathbb{R}^{N}} \chi\left(\varepsilon z+a^{i}\right)|w(z)|^{p} d z}{\int_{\mathbb{R}^{N}}|w(z)|^{p} d z} \\
& \rightarrow a^{i} \quad \text { as } \varepsilon \rightarrow 0^{+},
\end{aligned}
$$

there exists $\varepsilon_{1}>0$ such that

$$
Q_{\varepsilon}\left(t_{\varepsilon}^{i} \sqrt{\alpha} w_{\varepsilon}^{i}, t_{\varepsilon}^{i} \sqrt{\beta} w_{\varepsilon}^{i}\right) \in \mathbf{K}_{\rho_{0} / 2} \quad \text { for any } \varepsilon \in\left(0, \varepsilon_{1}\right) \text { and each } 1 \leq i \leq k .
$$

We need the following lemmas to prove that $\beta_{\lambda, \mu}^{i}<\widetilde{\beta}_{\lambda, \mu}^{i}$ for sufficiently small $\varepsilon, \lambda, \mu$.

Lemma 4.3 $\theta_{\max }=\frac{p-2}{2 p}\left(S_{\alpha, \beta}\right)^{p /(p-2)}$.

Proof From Part I of Lemma 4.1, we obtain $\sup _{t \geq 0} I_{\max }\left(t \sqrt{\alpha} w_{\varepsilon}^{i}, t \sqrt{\beta} w_{\varepsilon}^{i}\right)=\frac{p-2}{2 p}\left(S_{\alpha, \beta}\right)^{p /(p-2)}$ uniformly in $i$. Similarly to Lemma 2.4 , there is a sequence $\left\{s_{\max }^{i}\right\} \subset \mathbb{R}^{+}$such that $\left(s_{\max }^{i} \sqrt{\alpha} w_{\varepsilon}^{i}, s_{\max }^{i} \sqrt{\beta} w_{\varepsilon}^{i}\right) \in \mathbf{N}_{\max }$ and

$$
\theta_{\max } \leq I_{\max }\left(s_{\max }^{i} \sqrt{\alpha} u_{\varepsilon}^{i}, s_{\max }^{i} \sqrt{\beta} u_{\varepsilon}^{i}\right)=\sup _{t \geq 0} J_{\max }\left(t \sqrt{\alpha} u_{\varepsilon}^{i}, t \sqrt{\beta} u_{\varepsilon}^{i}\right)=\frac{p-2}{2 p}\left(S_{\alpha, \beta}\right)^{p /(p-2)} .
$$

Let $\left\{\left(u_{n}, v_{n}\right)\right\} \subset \mathbf{N}_{\max }$ be a minimizing sequence of $\theta_{\max }$ for $I_{\max }$. It follows that $\left\|\left(u_{n}, v_{n}\right)\right\|_{H}^{2}=$ $\int_{\mathbb{R}^{N}}\left|u_{n}\right|^{\alpha}\left|v_{n}\right|^{\beta} d z$ and

$$
\begin{aligned}
\theta_{\max } & =\frac{1}{2}\left\|\left(u_{n}, v_{n}\right)\right\|_{H}^{2}-\frac{1}{p} \int_{\mathbb{R}^{N}}\left|u_{n}\right|^{\alpha}\left|v_{n}\right|^{\beta} d z+o_{n}(1) \\
& =\frac{p-2}{2 p}\left\|\left(u_{n}, v_{n}\right)\right\|_{H}^{2}+o_{n}(1) .
\end{aligned}
$$

We may assume that $\left\|\left(u_{n}, v_{n}\right)\right\|_{H}^{2} \rightarrow l$ and $\int_{\mathbb{R}^{N}}\left|u_{n}\right|^{\alpha}\left|v_{n}\right|^{\beta} d z \rightarrow l$ as $n \rightarrow \infty$, where $l=$ $\frac{2 p}{p-2} \theta_{\max }>0$. By the definition of $S_{\alpha, \beta}$, then $S_{\alpha, \beta} l^{\frac{2}{p}} \leq l$. We can deduce that $S_{\alpha, \beta} \leq l^{\frac{p-2}{p}}=$ $\left(\frac{2 p}{p-2} \theta_{\max }\right)^{\frac{p-2}{p}}$, that is, $\frac{p-2}{2 p}\left(S_{\alpha, \beta}\right)^{p /(p-2)} \leq \theta_{\max }$.

Lemma 4.4 There exists a number $\delta_{0}>0$ such that if $(u, v) \in \mathbf{N}_{\varepsilon}$ and $I_{\varepsilon}(u, v) \leq \theta_{\max }+\delta_{0}$, then $Q_{\varepsilon}(u, v) \in \mathbf{K}_{\rho_{0} / 2}$ for any $0<\varepsilon<\varepsilon_{1}$.

Proof On the contrary, there exist the sequences $\left\{\varepsilon_{n}\right\} \subset \mathbb{R}^{+}$and $\left\{\left(u_{n}, v_{n}\right)\right\} \subset \mathbf{N}_{\varepsilon_{n}}$ such that $\varepsilon_{n} \rightarrow 0, I_{\varepsilon_{n}}\left(u_{n}, v_{n}\right)=\theta_{\max }(>0)+o_{n}(1)$ as $n \rightarrow \infty$ and $Q_{\varepsilon_{n}}\left(u_{n}, v_{n}\right) \notin \mathbf{K}_{\rho_{0} / 2}$ for all $n \in \mathbb{N}$. It is easy to check that $\left\{\left(u_{n}, v_{n}\right)\right\}$ is bounded in $H$. Suppose that $\int_{\mathbb{R}^{N}}\left|u_{n}\right|^{\alpha}\left|v_{n}\right|^{\beta} d z \rightarrow 0$ as $n \rightarrow \infty$. Since

$$
\left\|\left(u_{n}, v_{n}\right)\right\|_{H}^{2}=\int_{\mathbb{R}^{N}} f\left(\varepsilon_{n} z\right)\left|u_{n}\right|^{\alpha}\left|v_{n}\right|^{\beta} d z \quad \text { for each } n \in \mathbb{N},
$$

then

$$
\theta_{\max }+o_{n}(1)=I_{\varepsilon_{n}}\left(u_{n}, v_{n}\right)=\left(\frac{1}{2}-\frac{1}{p}\right) \int_{\mathbb{R}^{N}} f\left(\varepsilon_{n} z\right)\left|u_{n}\right|^{\alpha}\left|v_{n}\right|^{\beta} d z \leq o_{n}(1)
$$


which is a contradiction. Thus, $\int_{\mathbb{R}^{N}}\left|u_{n}\right|^{\alpha}\left|v_{n}\right|^{\beta} d z \nrightarrow 0$ as $n \rightarrow \infty$. Similarly to the concentration-compactness principle (see Lions $[15,16]$ or Wang $[6$, Lemma 2.16]), then there exist a constant $c_{0}>0$ and a sequence $\left\{\widetilde{z_{n}}\right\} \subset \mathbb{R}^{N}$ such that

$$
\int_{B^{N}\left(\tilde{z}_{n} ; 1\right)}\left|u_{n}\right|^{\frac{\alpha l}{p}}\left|v_{n}\right|^{\frac{\beta l}{p}} d z \geq c_{0}>0
$$

where $2<l<p=\alpha+\beta<2^{*}$ and $p=l(1-t)+2^{*} t$ for some $t \in((N-2) / N, 1)$. Let $\left(\widetilde{u_{n}}(z), \widetilde{v_{n}}(z)\right)=\left(u_{n}\left(z+\widetilde{z_{n}}\right), v_{n}\left(z+\widetilde{z_{n}}\right)\right)$. Then there are a subsequence $\left\{\left(\widetilde{u_{n}}, \widetilde{v_{n}}\right)\right\}$ and $(\widetilde{u}, \widetilde{v}) \in$ $H$ such that $\widetilde{u_{n}} \rightarrow \widetilde{u}$ and $\widetilde{v_{n}} \rightarrow \widetilde{v}$ weakly in $H^{1}\left(\mathbb{R}^{N}\right)$. Using the similar computation of Lemma 2.4, there is a sequence $\left\{s_{\max }^{n}\right\} \subset \mathbb{R}^{+}$such that $\left(s_{\max }^{n} \tilde{u_{n}}, s_{\max }^{n} \widetilde{x_{n}}\right) \in \mathbf{N}_{\max }$ and

$$
\begin{aligned}
0 & <\theta_{\max } \leq I_{\max }\left(s_{\max }^{n} \widetilde{u_{n}}, s_{\max }^{n} \widetilde{v_{n}}\right)=I_{\max }\left(s_{\max }^{n} u_{n}, s_{\max }^{n} v_{n}\right) \\
& \leq I_{\varepsilon_{n}}\left(s_{\max }^{n} u_{n}, s_{\max }^{n} v_{n}\right) \leq I_{\varepsilon_{n}}\left(u_{n}, v_{n}\right)=\theta_{\max }+o_{n}(1) \quad \text { as } n \rightarrow \infty .
\end{aligned}
$$

We deduce that a subsequence $\left\{s_{\max }^{n}\right\}$ satisfies $s_{\max }^{n} \rightarrow s_{0}>0$. Then there are a subsequence $\left\{\left(s_{\max }^{n} \tilde{u_{n}}, s_{\max }^{n} \tilde{v}_{n}\right)\right\}$ and $\left(s_{0} \widetilde{u}, s_{0} \widetilde{v}\right) \in H$ such that $s_{\max }^{n} \tilde{u_{n}} \rightarrow s_{0} \tilde{u}$ and $s_{\max }^{n} \widetilde{v_{n}} \rightarrow s_{0} \widetilde{v}$ weakly in $H^{1}\left(\mathbb{R}^{N}\right)$. By (4.3), then $\widetilde{u} \neq 0$ and $\widetilde{v} \neq 0$. Applying Ekeland's variational principle, there exists a $(\mathrm{PS})_{\theta_{\max }}$-sequence $\left\{\left(U_{n}, V_{n}\right)\right\}$ for $I_{\max }$ and $\left\|\left(U_{n}-s_{\max }^{n} \tilde{u_{n}}, V_{n}-s_{\max }^{n}{\tilde{v_{n}}}_{n}\right)\right\|_{H}=o_{n}(1)$. Similarly to the proof of Lemma 3.3, there exist a subsequence $\left\{\left(U_{n}, V_{n}\right)\right\}$ and $\left(U_{0}, V_{0}\right) \in H$ such that $U_{n} \rightarrow U_{0}, V_{n} \rightarrow V_{0}$ strongly in $H^{1}\left(\mathbb{R}^{N}\right)$ and $I_{\max }\left(U_{0}, V_{0}\right)=\theta_{\max }$. Now, we want to show that there exists a subsequence $\left\{z_{n}\right\}=\left\{\varepsilon_{n} \widetilde{z_{n}}\right\}$ such that $z_{n} \rightarrow z_{0} \in \mathbf{K}$.

(i) Claim that the sequence $\left\{z_{n}\right\}$ is bounded in $\mathbb{R}^{N}$. On the contrary, assume that $\left|z_{n}\right| \rightarrow \infty$, then

$$
\begin{aligned}
\theta_{\max } & =I_{\max }\left(U_{0}, V_{0}\right)<\frac{1}{2}\left\|\left(U_{0}, V_{0}\right)\right\|_{H}^{2}-\frac{1}{p} \int_{\mathbb{R}^{N}} f_{\infty}\left|U_{0}\right|^{\alpha}\left|V_{0}\right|^{\beta} d z \\
& \leq \liminf _{n \rightarrow \infty}\left[\frac{\left(s_{\max }^{n}\right)^{2}}{2}\left\|\left(\widetilde{u_{n}}, \widetilde{v_{n}}\right)\right\|_{H}^{2}-\frac{\left(s_{\max }^{n}\right)^{p}}{p} \int_{\mathbb{R}^{N}} f\left(\varepsilon_{n} z+z_{n}\right)\left|\widetilde{u_{n}}\right|^{\alpha}\left|\widetilde{v_{n}}\right|^{\beta} d z\right] \\
& =\liminf _{n \rightarrow \infty}\left[\frac{\left(s_{\max }^{n}\right)^{2}}{2}\left\|\left(u_{n}, v_{n}\right)\right\|_{H}^{2}-\frac{\left(s_{\max }^{n}\right)^{p}}{p} \int_{\mathbb{R}^{N}} f\left(\varepsilon_{n} z\right)\left|u_{n}\right|^{\alpha}\left|v_{n}\right|^{\beta} d z\right] \\
& =\liminf _{n \rightarrow \infty} I_{\varepsilon_{n}}\left(s_{\max }^{n} u_{n}, s_{\max }^{n} v_{n}\right) \leq \liminf _{n \rightarrow \infty} I_{\varepsilon_{n}}\left(u_{n}, v_{n}\right)=\theta_{\max },
\end{aligned}
$$

which is a contradiction.

(ii) Claim that $z_{0} \in \mathbf{K}$. On the contrary, assume that $z_{0} \notin \mathbf{K}$, that is, $f\left(z_{0}\right)<1=$ $\max _{z \in \mathbb{R}^{N}} f(z)$. Then use the argument of (i) to obtain that

$$
\begin{aligned}
\theta_{\max } & =I_{\max }\left(U_{0}, V_{0}\right) \leq I_{\max }\left(s_{0} U_{0}, s_{0} V_{0}\right) \\
& <\frac{\left(s_{0}\right)^{2}}{2}\left\|\left(U_{0}, V_{0}\right)\right\|_{H}^{2}-\frac{\left(s_{0}\right)^{p}}{p} \int_{\mathbb{R}^{N}} f\left(z_{0}\right)\left|U_{0}\right|^{\alpha}\left|V_{0}\right|^{\beta} d z \\
& \leq \liminf _{n \rightarrow \infty}\left[\frac{\left(s_{\max }^{n}\right)^{2}}{2}\left\|\left(\widetilde{u_{n}}, \widetilde{v_{n}}\right)\right\|_{H}^{2}-\frac{\left(s_{\max }^{n}\right)^{p}}{p} \int_{\mathbb{R}^{N}} f\left(\varepsilon_{n} z+z_{n}\right)\left|\widetilde{u_{n}}\right|^{\alpha}\left|\widetilde{v_{n}}\right|^{\beta} d z\right] \\
& \leq \theta_{\max },
\end{aligned}
$$

which is a contradiction. 
Since $\left\|\left(U_{n}-s_{\max }^{n} \tilde{u_{n}}, V_{n}-s_{\max }^{n} \widetilde{x_{n}}\right)\right\|_{H}=o_{n}(1)$ and $U_{n} \rightarrow U_{0}, V_{n} \rightarrow V_{0}$ strongly in $H^{1}\left(\mathbb{R}^{N}\right)$, we have that

$$
\begin{aligned}
Q_{\varepsilon_{n}}\left(u_{n}, v_{n}\right) & =\frac{\int_{\mathbb{R}^{N}} \chi\left(\varepsilon_{n} z\right)\left|\widetilde{u_{n}}\left(z-\widetilde{z_{n}}\right)\right|^{\alpha}\left|\widetilde{v_{n}}\left(z-\widetilde{z_{n}}\right)\right|^{\beta} d z}{\int_{\mathbb{R}^{N}}\left|\widetilde{u_{n}}\left(z-\widetilde{z_{n}}\right)\right|^{\alpha}\left|\widetilde{v_{n}}\left(z-\widetilde{z_{n}}\right)\right|^{\beta} d z} \\
& =\frac{\int_{\mathbb{R}^{N}} \chi\left(\varepsilon_{n} z+\varepsilon_{n} \widetilde{z_{n}}\right)\left|U_{0}\right|^{\alpha}\left|V_{0}\right|^{\beta} d z}{\int_{\mathbb{R}^{N}}\left|U_{0}\right|^{\alpha}\left|V_{0}\right|^{\beta} d z} \rightarrow z_{0} \in \mathbf{K}_{\rho_{0} / 2} \quad \text { as } n \rightarrow \infty,
\end{aligned}
$$

which is a contradiction.

Hence, there exists $\delta_{0}>0$ such that if $(u, v) \in \mathbf{N}_{\varepsilon}$ and $I_{\varepsilon}(u, v) \leq \theta_{\max }+\delta_{0}$, then $Q_{\varepsilon}(u, v) \in$ $\mathbf{K}_{\rho_{0} / 2}$ for any $0<\varepsilon<\varepsilon_{1}$.

Lemma 4.5 If $(u, v) \in \mathbf{M}_{\varepsilon, \lambda, \mu}$ and $J_{\varepsilon, \lambda, \mu}(u, v) \leq \theta_{\max }+\delta_{0} / 2$, then there exists a number $\Lambda^{*}>0$ such that $Q_{\varepsilon}(u, v) \in \mathbf{K}_{\rho_{0} / 2}$ for any $0<\varepsilon<\varepsilon_{1}$ and $0<\lambda+\mu<\Lambda^{*}$.

Proof Using the similar computation in Lemma 2.4, we obtain that there is the unique positive number

$$
s_{\varepsilon}=\left(\frac{\|(u, v)\|_{H}^{2}}{\int_{\mathbb{R}^{N}} f(\varepsilon z)|u|^{\alpha}|v|^{\beta} d z}\right)^{1 /(p-2)}
$$

such that $\left(s_{\varepsilon} u, s_{\varepsilon} \nu\right) \in \mathbf{N}_{\varepsilon}$. We want to show that there exists $\Lambda_{0}>0$ such that if $0<\lambda+\mu<$ $\Lambda_{0}$, then $s_{\varepsilon}<c$ for some constant $c>0$ (independent of $u$ and $v$ ). First, for $(u, v) \in \mathbf{M}_{\varepsilon, \lambda, \mu}$,

$$
0<d_{0} \leq \theta_{\varepsilon, \lambda, \mu} \leq J_{\varepsilon, \lambda, \mu}(u, v) \leq \theta_{\max }+\delta_{0} / 2 .
$$

Since $\left\langle J_{\varepsilon, \lambda, \mu}^{\prime}(u, v),(u, v)\right\rangle=0$, then

$$
\begin{aligned}
\theta_{\max }+\delta_{0} / 2 & \geq J_{\varepsilon, \lambda, \mu}(u, v) \\
& =\left(\frac{1}{2}-\frac{1}{q}\right)\|(u, v)\|_{H}^{2}+\left(\frac{1}{q}-\frac{1}{p}\right) \int_{\mathbb{R}^{N}} f(\varepsilon z)|u|^{\alpha}|v|^{\beta} d z \\
& \geq \frac{q-2}{2 q}\|(u, v)\|_{H}^{2}, \quad \text { that is, }\|(u, v)\|_{H}^{2} \leq c_{1}=\frac{2 q}{q-2}\left(\theta_{\max }+\delta_{0} / 2\right),
\end{aligned}
$$

and

$$
\begin{aligned}
d_{0} & \leq J_{\varepsilon, \lambda, \mu}(u, v) \\
& =\left(\frac{1}{2}-\frac{1}{p}\right)\|(u, v)\|_{H}^{2}-\left(\frac{1}{q}-\frac{1}{p}\right) \int_{\Omega}\left(\lambda g(\varepsilon z)|u|^{q}+\mu h(\varepsilon z)|v|^{q}\right) d z \\
& \leq \frac{p-2}{2 p}\|(u, v)\|_{H}^{2}, \quad \text { that is, } \quad\|(u, v)\|_{H}^{2} \geq c_{2}=\frac{2 p}{p-2} d_{0} .
\end{aligned}
$$

Moreover, we have that

$$
\begin{aligned}
\int_{\Omega} f(\varepsilon z)|u|^{\alpha}|v|^{\beta} d z & =\|(u, v)\|_{H}^{2}-\int_{\mathbb{R}^{N}}\left(\lambda g(\varepsilon z)|u|^{q}+\mu h(\varepsilon z)|v|^{q}\right) d z \\
& \geq c_{2}-\operatorname{Max} S_{p}^{-\frac{q}{2}}(\lambda+\mu) c_{1}^{q / 2}
\end{aligned}
$$


where Max $=\max \left\{\|g\|_{m},\|h\|_{m}\right\}$. It follows that there exists $\Lambda_{0}>0$ such that for $0<\lambda+$ $\mu<\Lambda_{0}$

$$
\int_{\mathbb{R}^{N}} f(\varepsilon z)|u|^{\alpha}|v|^{\beta} d z \geq c_{2}-\operatorname{Max}_{p}^{-\frac{q}{2}}(\lambda+\mu)\left(c_{1}\right)^{q / 2}>0 .
$$

Hence, by (4.4), (4.5) and (4.6), $s_{\varepsilon}<c$ for some constant $c>0$ (independent of $u$ and $v$ ) for $0<\lambda+\mu<\Lambda_{0}$. Now, we obtain that

$$
\begin{aligned}
\theta_{\max }+\delta_{0} / 2 \geq & J_{\varepsilon, \lambda, \mu}(u, v)=\sup _{t \geq 0} J_{\varepsilon, \lambda, \mu}(t u, t v) \geq J_{\varepsilon, \lambda, \mu}\left(s_{\varepsilon} u, s_{\varepsilon} v\right) \\
= & \frac{1}{2}\left\|\left(s_{\varepsilon} u, s_{\varepsilon} v\right)\right\|_{H}^{2}-\frac{1}{p} \int_{\mathbb{R}^{N}} f(\varepsilon z)\left|s_{\varepsilon} u\right|^{\alpha}\left|s_{\varepsilon} v\right|^{\beta} d z \\
& -\frac{1}{q} \int_{\mathbb{R}^{N}}\left(\lambda g(\varepsilon z)\left|s_{\varepsilon} u\right|^{q}+\mu h(\varepsilon z)\left|s_{\varepsilon} v\right|^{q}\right) d z \\
\geq & I_{\varepsilon}\left(s_{\varepsilon} u, s_{\varepsilon} v\right)-\frac{1}{q} \int_{\mathbb{R}^{N}}\left(\lambda g(\varepsilon z)\left|s_{\varepsilon} u\right|^{q}+\mu h(\varepsilon z)\left|s_{\varepsilon} v\right|^{q}\right) d z .
\end{aligned}
$$

From the above inequality, we deduce that for any $0<\varepsilon<\varepsilon_{1}$ and $0<\lambda+\mu<\Lambda_{0}$,

$$
\begin{aligned}
I_{\varepsilon}\left(s_{\varepsilon} u, s_{\varepsilon} v\right) & \leq \theta_{\max }+\delta_{0} / 2+\frac{1}{q} \int_{\mathbb{R}^{N}}\left(\lambda g(\varepsilon z)\left|s_{\varepsilon} u\right|^{q}+\mu h(\varepsilon z)\left|s_{\varepsilon} v\right|^{q}\right) d z \\
& \leq \theta_{\max }+\delta_{0} / 2+\operatorname{Max}(\lambda+\mu) S_{p}^{-\frac{q}{2}}\left\|\left(s_{\varepsilon} u, s_{\varepsilon} v\right)\right\|_{H}^{q} \\
& <\theta_{\max }+\delta_{0} / 2+\operatorname{Max} S_{p}^{-\frac{q}{2}}(\lambda+\mu) c^{q}\left(c_{1}\right)^{q / 2} .
\end{aligned}
$$

Hence, there exists $\Lambda^{*} \in\left(0, \Lambda_{0}\right)$ such that for $0<\lambda+\mu<\Lambda^{*}$,

$$
I_{\varepsilon}\left(s_{\varepsilon} u, s_{\varepsilon} v\right) \leq \theta_{\max }+\delta_{0}, \quad \text { where }\left(s_{\varepsilon} u, s_{\varepsilon} v\right) \in \mathbf{N}_{\varepsilon} .
$$

By Lemma 4.4, we obtain

$$
Q_{\varepsilon}\left(s_{\varepsilon} u, s_{\varepsilon} v\right)=\frac{\int_{\mathbb{R}^{N}} \chi(\varepsilon z)\left|s_{\varepsilon} u\right|^{\alpha}\left|s_{\varepsilon} \nu\right|^{\beta} d z}{\int_{\mathbb{R}^{N}}\left|s_{\varepsilon} u\right|^{\alpha}\left|s_{\varepsilon} v\right|^{\beta} d z} \in \mathbf{K}_{\rho_{0} / 2}
$$

or $Q_{\varepsilon}(u, v) \in \mathbf{K}_{\rho_{0} / 2}$ for any $0<\varepsilon<\varepsilon_{0}$ and $0<\lambda+\mu<\Lambda^{*}$.

Since $f_{\infty}<1$, then by Lemma 4.3,

$$
\theta_{\max }=\frac{p-2}{2 p}\left(S_{\alpha, \beta}\right)^{p /(p-2)}<\frac{p-2}{2 p} \frac{\left(S_{\alpha, \beta}\right)^{p /(p-2)}}{\left(f_{\infty}\right)^{2 /(p-2)}} .
$$

By Lemmas 4.1, 4.2 and (4.7), for any $0<\varepsilon<\varepsilon_{0}\left(<\varepsilon_{1}\right)$ and $0<\lambda+\mu<\Lambda^{*}$,

$$
\beta_{\varepsilon, \lambda, \mu}^{i} \leq J_{\varepsilon, \lambda, \mu}\left(t_{\varepsilon}^{i} \sqrt{\alpha} w_{\varepsilon}^{i}, t_{\varepsilon}^{i} \sqrt{\beta} w_{\varepsilon}^{i}\right)<\frac{p-2}{2 p} \frac{\left(S_{\alpha, \beta}\right)^{p /(p-2)}}{\left(f_{\infty}\right)^{2 /(p-2)}} .
$$

Applying above Lemma 4.5, we get that

$$
\widetilde{\beta}_{\varepsilon, \lambda, \mu}^{i} \geq \theta_{\max }+\delta_{0} / 2 \quad \text { for any } 0<\varepsilon<\varepsilon_{0} \text { and } 0<\lambda+\mu<\Lambda^{*} .
$$


For each $1 \leq i \leq k$, by (4.8) and (4.9), we obtain that

$$
\beta_{\varepsilon, \lambda, \mu}^{i}<\widetilde{\beta}_{\varepsilon, \lambda, \mu}^{i} \text { for any } 0<\varepsilon<\varepsilon_{0} \text { and } 0<\lambda+\mu<\Lambda^{*}
$$

It follows that

$$
\beta_{\varepsilon, \lambda, \mu}^{i}=\inf _{(u, v) \in O_{\varepsilon, \lambda, \mu}^{i} \cup \partial O_{\varepsilon, \lambda, \mu}^{i}} J_{\varepsilon, \lambda, \mu}(u, v) \text { for any } 0<\varepsilon<\varepsilon_{0} \text { and } 0<\lambda+\mu<\Lambda^{*} .
$$

Then applying Ekeland's variational principle and using the standard computation, we have the following lemma.

Lemma 4.6 For each $1 \leq i \leq k$, there is a $(P S)_{\beta_{\varepsilon, \lambda, \mu}^{i}}$-sequence $\left\{\left(u_{n}, v_{n}\right)\right\} \subset O_{\varepsilon, \lambda, \mu}^{i}$ in $H$ for $J_{\varepsilon, \lambda, \mu}$.

Proof See Cao-Zhou [8].

Proof of Theorem 1.2 For any $0<\varepsilon<\varepsilon_{0}$ and $0<\lambda+\mu<\Lambda^{*}$, by Lemma 4.6, there is a (PS) $\beta_{\varepsilon, \lambda, \mu}^{i}$-sequence $\left\{\left(u_{n}, v_{n}\right)\right\} \subset O_{\varepsilon, \lambda, \mu}^{i}$ for $J_{\varepsilon, \lambda, \mu}$ where $1 \leq i \leq k$. By (4.8), we obtain that

$$
\beta_{\varepsilon, \lambda, \mu}^{i}<\frac{p-2}{2 p} \frac{\left(S_{\alpha, \beta}\right)^{p /(p-2)}}{\left(f_{\infty}\right)^{2 /(p-2)}} .
$$

Since $J_{\varepsilon, \lambda, \mu}$ satisfies the (PS) ${ }_{\gamma}$-condition for $\gamma \in\left(-\infty, \frac{p-2}{2 p} \frac{\left(S_{\alpha, \beta}\right)^{p /(p-2)}}{\left(f_{\infty}\right)^{2 /(p-2)}}\right)$, then $J_{\varepsilon, \lambda, \mu}$ has at least $k$ critical points in $\mathbf{M}_{\varepsilon, \lambda, \mu}$ for any $0<\varepsilon<\varepsilon_{0}$ and $0<\lambda+\mu<\Lambda^{*}$. Set $u_{+}=\max \{u, 0\}$ and $\nu_{+}=\max \{v, 0\}$. Replace the terms $\int_{\mathbb{R}^{N}} f(\varepsilon z)|u|^{\alpha}|v|^{\beta} d z$ and $\int_{\mathbb{R}^{N}}\left(\lambda g(\varepsilon z)|u|^{q}+\mu h(\varepsilon z)|v|^{q}\right) d z$ of the functional $J_{\varepsilon, \lambda, \mu}$ by $\int_{\mathbb{R}^{N}} f(\varepsilon z) u_{+}^{\alpha} \nu_{+}^{\beta} d z$ and $\int_{\mathbb{R}^{N}}\left(\lambda g(\varepsilon z) u_{+}^{q}+\mu h(\varepsilon z) v_{+}^{q}\right) d z$, respectively. It follows that $\left(E_{\varepsilon, \lambda, \mu}\right)$ has $k$ nonnegative solutions. Applying the maximum principle, $\left(E_{\varepsilon, \lambda, \mu}\right)$ admits at least $k$ positive solutions.

\section{Competing interests}

The author declares that he has no competing interests.

\section{Acknowledgements}

The author was grateful for the referee's helpful suggestions and comments.

Received: 29 March 2012 Accepted: 4 October 2012 Published: 24 October 2012

\section{References}

1. Lin, TC, Wei, J: Spikes in two coupled nonlinear Schrödinger equations. Ann. Inst. Henri Poincaré, Anal. Non Linéaire 22, 403-439 (2005)

2. Alves, CO, de Morais Filho, DC, Souto, MAS: On systems of elliptic equations involving subcritical or critical Sobolev exponents. Nonlinear Anal. 42, 771-787 (2000)

3. Han, P: Multiple positive solutions of nonhomogeneous elliptic system involving critical Sobolev exponents. Nonlinear Anal. 64, 869-886 (2006)

4. Hsu, TS: Multiple positive solutions for a critical quasilinear elliptic system with concave-convex nonlinearities. Nonlinear Anal. 71, 2688-2698 (2009)

5. Wu, TF: The Nehari manifold for a semilinear elliptic system involving sign-changing weight function. Nonlinear Anal. 68, 1733-1745 (2008)

6. Wang, HC: Palais-Smale approaches to semilinear elliptic equations in unbounded domains. Electron. J. Differ. Equ. Monograph 06 (2004)

7. Han, P: The effect of the domain topology on the number of positive solutions of elliptic systems involving critical Sobolev exponents. Houst. J. Math. 32, 1241-1257 (2006)

8. Cao, DM, Zhou, HS: Multiple positive solutions of nonhomogeneous semilinear elliptic equations in $\mathbb{R}^{N}$. Proc. R. Soc. Edinb., Sect. A, Math. 126, 443-463 (1996) 
9. Tarantello, G: On nonhomogeneous elliptic involving critical Sobolev exponent. Ann. Inst. Henri Poincaré, Anal. Non Linéaire 9, 281-304 (1992)

10. Bahri, A, Li, YY: On a min-max procedure for the existence of a positive solution for certain scalar field equations in $\mathbb{R}^{N}$. Rev. Mat. Iberoam. 6, 1-15 (1990)

11. Bahri, A, Lions, PL: On the existence of a positive solution of semilinear elliptic equations in unbounded domains. Ann. Inst. Henri Poincaré, Anal. Non Linéaire 14, 365-413 (1997)

12. Gidas, B, Ni, WM, Nirenberg, L: Symmetry and related properties via the maximum principle. Commun. Math. Phys. 68, 209-243 (1979)

13. Kwong, MK: Uniqueness of positive solutions of $\Delta u-u+u^{p}=0$ in $\mathbb{R}^{N}$. Arch. Ration. Mech. Anal. 105, 234-266 (1989)

14. Lien, WC, Tzeng, SY, Wang, HC: Existence of solutions of semilinear elliptic problems on unbounded domains. Differ. Integral Equ. 6, 1281-1298 (1993)

15. Lions, PL: The concentration-compactness principle in the calculus of variations. The locally compact case. I. Ann. Inst. Henri Poincaré, Anal. Non Linéaire 1, 109-145 (1984)

16. Lions, PL: The concentration-compactness principle in the calculus of variations. The locally compact case. II. Ann. Inst. Henri Poincaré, Anal. Non Linéaire 1, 223-283 (1984)

doi:10.1186/1687-2770-2012-118

Cite this article as: Lin: Multiple positive solutions for semilinear elliptic systems involving subcritical nonlinearities in $\mathbb{R}^{N}$. Boundary Value Problems 2012 2012:118.

\section{Submit your manuscript to a SpringerOpen ${ }^{\odot}$ journal and benefit from:}

- Convenient online submission

Rigorous peer review

- Immediate publication on acceptance

- Open access: articles freely available online

- High visibility within the field

- Retaining the copyright to your article 